\title{
Demographic, multi-morbidity and genetic impact on myocardial involvement and its recovery from COVID-19: protocol design of COVID-HEART—a UK, multicentre, observational study
}

Miroslawa Gorecka', Gerry P. McCann², Colin Berry ${ }^{3}$, Vanessa M. Ferreira ${ }^{4}$, James C. Moon Christopher A. Miller ${ }^{6}$, Amedeo Chiribiri ${ }^{7}$, Sanjay Prasad ${ }^{8}$, Marc R. Dweck ${ }^{9}$, Chiara Bucciarelli-Ducci ${ }^{10}$, Dana Dawson ${ }^{11}$, Marianna Fontana ${ }^{12}$, Peter W. Macfarlane ${ }^{13}$, Alex McConnachie ${ }^{14}$, Stefan Neubauer ${ }^{4}$ and John P. Greenwood ${ }^{1 *}$ (D) on behalf of the COVID-HEART investigators

\begin{abstract}
Background: Although coronavirus disease 2019 (COVID-19) is primarily a respiratory illness, myocardial injury is increasingly reported and associated with adverse outcomes. However, the pathophysiology, extent of myocardial injury and clinical significance remains unclear.
\end{abstract}

Methods: COVID-HEART is a UK, multicentre, prospective, observational, longitudinal cohort study of patients with confirmed COVID-19 and elevated troponin (sex-specific > 99th centile). Baseline assessment will be whilst recovering in-hospital or recently discharged, and include cardiovascular magnetic resonance (CMR) imaging, quality of life (QoL) assessments, electrocardiogram (ECG), serum biomarkers and genetics. Assessment at 6-months includes repeat CMR, QoL assessments and 6-min walk test (6MWT). The CMR protocol includes cine imaging, T1/T2 mapping, aortic distensibility, late gadolinium enhancement (LGE), and adenosine stress myocardial perfusion imaging in selected patients. The main objectives of the study are to: (1) characterise the extent and nature of myocardial involvement in COVID-19 patients with an elevated troponin, (2) assess how cardiac involvement and clinical outcome associate with recognised risk factors for mortality (age, sex, ethnicity and comorbidities) and genetic factors, (3) evaluate if differences in myocardial recovery at 6 months are dependent on demographics, genetics and comorbidities, (4) understand the impact of recovery status at 6 months on patient-reported QoL and functional capacity.

Discussion: COVID-HEART will provide detailed characterisation of cardiac involvement, and its repair and recovery in relation to comorbidity, genetics, patient-reported QoL measures and functional capacity.

Clinical Trial registration: ISRCTN 58667920. Registered 04 August 2020.

*Correspondence: j.greenwood@leeds.ac.uk

${ }^{1}$ Institute of Cardiovascular and Metabolic Medicine, University of Leeds, Leeds LS2 9JT, UK

Full list of author information is available at the end of the article

(c) The Author(s) 2021. Open Access This article is licensed under a Creative Commons Attribution 4.0 International License, which permits use, sharing, adaptation, distribution and reproduction in any medium or format, as long as you give appropriate credit to the original author(s) and the source, provide a link to the Creative Commons licence, and indicate if changes were made. The images or other third party material in this article are included in the article's Creative Commons licence, unless indicated otherwise in a credit line to the material. If material is not included in the article's Creative Commons licence and your intended use is not permitted by statutory regulation or exceeds the permitted use, you will need to obtain permission directly from the copyright holder. To view a copy of this licence, visit http://creativecommons.org/licenses/by/4.0/. The Creative Commons Public Domain Dedication waiver (http://creativeco mmons.org/publicdomain/zero/1.0/) applies to the data made available in this article, unless otherwise stated in a credit line to the data. 
Keywords: COVID-19, Coronavirus, Cardiovascular magnetic resonance, Myocarditis, Myopericarditis, Myocardial infarction, myocardial injury, Myocardial inflammation, Myocardial repair, Cardiovascular disease

\section{Background}

The coronavirus disease 2019 (COVID-19) severe acute respiratory syndrome coronavirus 2 (SARS-CoV-2) pandemic [1] was initially recognised as a primarily respiratory illness with a severe acute respiratory syndrome, but it is now known that it can affect multiple organs with a wide spectrum of disease severity $[2,3]$.

The direct cardiovascular impact of COVID-19 is three-fold. Firstly, underlying cardiovascular disease (CVD) can predispose patients to the infection and is associated with an increased illness severity. Secondly, COVID-19 may exacerbate underlying cardiovascular co-morbidities leading to symptom destabilisation and potentially acute admission to hospital. Thirdly, de novo cardiac complications of COVID-19 may occur [4], with SARS-CoV-2 infection being implicated in acute myocarditis, pericarditis, prothrombotic complications, left ventricular (LV) and/or right ventricular (RV) dysfunction, arrhythmia and ischaemic sequelae in the presence or absence of underlying coronary artery disease (CAD) [1, 4-6].

Multiple studies have demonstrated that myocardial injury, characterised by elevation in serum cardiac biomarkers, is common in COVID-19. The prevalence of myocardial injury, however, is variably reported, with early studies quoting a prevalence of $8-12 \%$ in hospitalised patients, whilst more recent data suggest that it is possibly much higher $[4,7]$. Subsequently, a larger multicentre study of 305 patients identified an elevated troponin in more than half of the hospitalised patients [8]. A recent German cohort study reported imaging evidence of myocardial injury defined by cardiovascular magnetic resonance (CMR) in over 70\%, although whether this was pre-existing or consequence was unclear with single time-point imaging [9]. The underlying mechanism of cardiac injury and troponin elevation remains unclear, but there are several potential explanations. Troponin $\mathrm{T}$ release is commonly seen in critically ill patients because of oxygen supply-demand mismatch and cytokine release, acute heart failure, RV strain (secondary to pneumonia, hypoxia and increased pulmonary artery pressure) and acute arrhythmia [10-12]. Furthermore, the acute-phase response to severe coronavirus infection can lead to coagulopathy and microvascular thrombosis, for example in the pulmonary and coronary circulations [13, 14]. Preexisting coronary risk factors, such as hypertension and diabetes also predispose patients with COVID-19 to myocardial infarction in the acute setting, possibly implicating it as another potential disease mechanism [15]. On a cellular level, however, direct myocardial injury may occur due to viral angiotensin-converting enzyme- 2 transmembrane receptor mediated damage, microvascular dysfunction or increased vessel wall permeability [16].

Troponin elevation in hospitalised COVID-19 patients is associated with higher morbidity and increased shortterm mortality $[4,8,17-21]$, particularly in those who also have underlying CVD [16]. Those with CV comorbidities (diabetes, hypertension, heart failure or CAD/ peripheral artery disease) are heavily over-represented in both severe COVID-19 and mortality [22, 23].

An elevated troponin should prompt a search for causation, and particularly imaging, as structural abnormalities are likely to be found in this cohort and further predict adverse outcomes (Fig. 1) [4, 8, 17-20, 24]. In patients with an elevated troponin and non-obstructive coronary arteries, CMR can provide the diagnosis in up to $90 \%$ of cases [25], and is the imaging modality of choice for diagnosis of acute myocarditis [26]. The diagnostic performance of the CMR-Lake Louise imaging criteria to identify acute myocarditis has been reported to have an overall diagnostic accuracy of around $83 \%$ (sensitivity $78-80 \%$ and specificity $87-88 \%)[27,28]$. CMR can also identify persistent inflammation and the presence of fibrosis in patients recovered from COVID-19 [9, 29], which may have long-term clinical implications. Furthermore, CMR is the reference standard method for assessing LV and RV pathology, whilst regional perfusion defects consistent with obstructive CAD can be accurately identified using CMR perfusion [30].

Based on the possibility that myocardial injury may be relatively common in COVID-19 patients, and has been shown to be a marker of poor prognosis, further studies are required to clarify the pathophysiology of SARS-CoV-2 related myocardial injury. In particular, the nature of injury may suggest the need for therapeutic intervention, which may be specific (e.g. antithrombotic, anti-inflammatory), or non-specific e.g. heart failure treatment. Importantly, the nature and extent of myocardial injury may also help risk stratify patients both acutely and chronically, and for this, multi-time-point imaging is required to separate out cause and effect. Finally, the underlying risk factors for myocardial involvement and the impact on long-term clinical outcomes need to be investigated. 


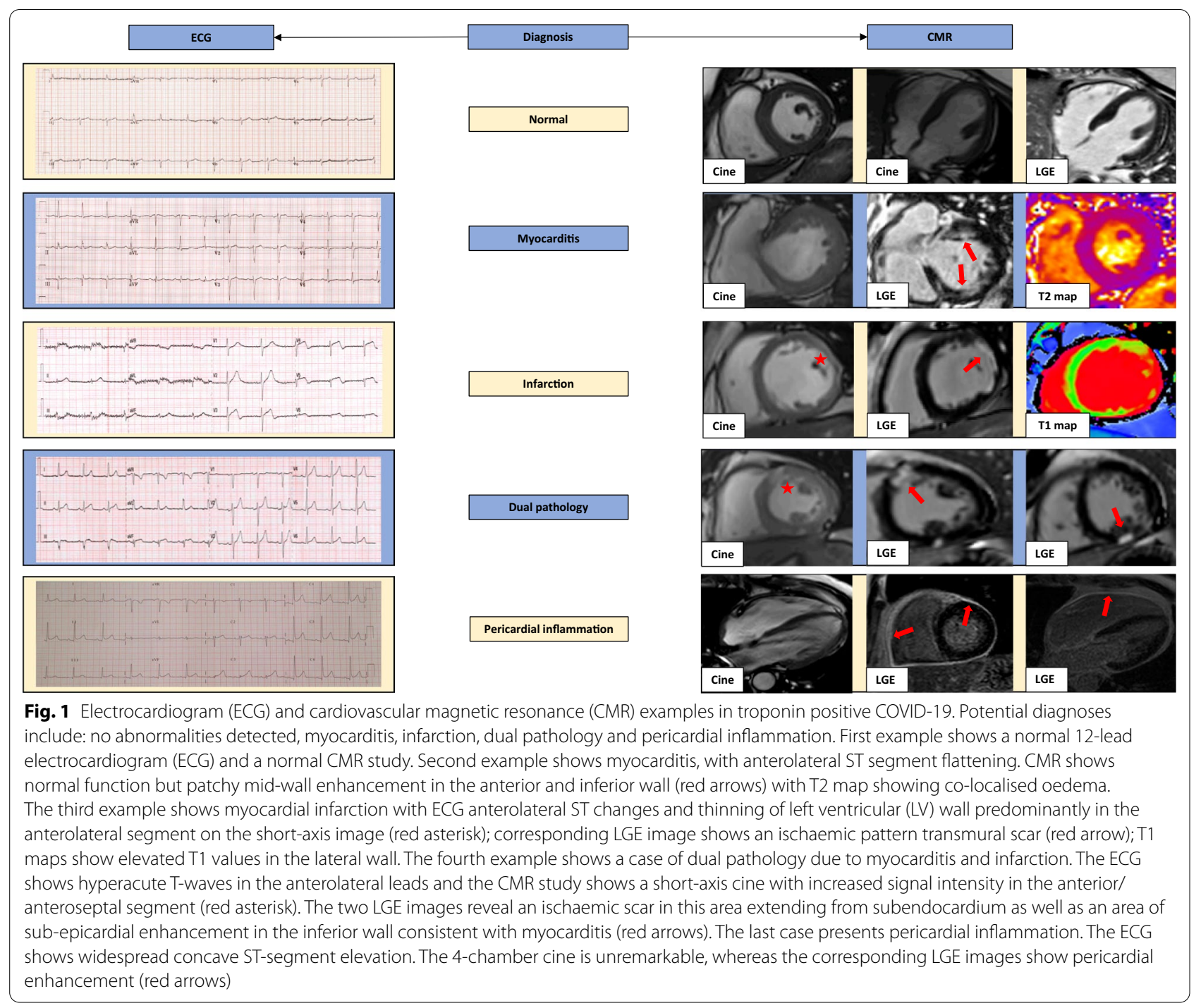

\section{Methods \\ Objectives}

The primary objectives of COVID-HEART are, for a hospitalised-recovering patient population (or those recently discharged), to: (1) characterise the nature and extent of myocardial involvement in COVID-19 patients with an elevated troponin; (2) assess how cardiac involvement and clinical outcome associate with recognised risk factors for mortality in COVID-19 (age, sex, ethnicity and comorbidities) and with genetic factors; (3) evaluate if differences in myocardial recovery at 6-months are dependent on demographics, genetics and CVD comorbidities (diabetes, hypertension, heart failure and CAD/ peripheral vascular disease); (4) understand the impact of recovery status at 6-months on patient-reported quality of life (QoL) and functional capacity.
The secondary objectives include: (a) to examine whether there are characteristic electrocardiogram (ECG) findings specific for SARS-CoV-2 myocarditis that are distinct from acute myocardial infarction which could minimise the requirement for invasive cardiac catheterisation and, (b) to establish the nature and prevalence of myocardial ischaemia and microvascular dysfunction (coronary vascular sub-study).

\section{Study design}

A longitudinal, multicentre, observational cohort study of UK patients presenting with COVID-19 and elevated serum troponin, who are either recovering in hospital or were recently discharged. 


\section{Ethics and registration}

Ethical approval for the COVID-HEART study has been granted by the UK National Research Ethics Service $(20 / \mathrm{NW} / 0292)$. COVID-HEART is registered on the 'International Standard Randomised Controlled Trial' registry (ISRCTN 5866,920) https://doi.org/10. 1186/ISRCTN58667920.

\section{Funding, sponsorship and prioritisation}

COVID-HEART is funded by the National Institute for Health Research (NIHR) and UK Research and Innovation (UKRI) COVID-19 Rapid Response Rolling Call (grant number COV0254), and sponsored by the University of Leeds, UK. The study has been endorsed by the British Society of Cardiovascular Magnetic Resonance (BSCMR) Research Group, and nationally prioritised, and received both BHF-NIHR Cardiovascular Partnership Flagship Status, and the NIHR Urgent Public Health Group identified it as an Urgent Public Health (UPH) study. Funding for the translation of the patient information leaflets into non-English languages was provided by the West Yorkshire and Humber Clinical Research Network (CV070).

\section{Study population}

Inclusion criteria include-hospitalised-recovering patient population (age $\geq 18$ years), or those recently discharged from hospital, with a diagnosis of COVID19 based upon either a pathology or radiology diagnosis, with cardiac biomarkers (troponin I or T) increased above the sex-specific upper reference limit of the local laboratory range. Exclusion criteria include being unable or unwilling to consent, contraindication to CMR, pregnancy or breast-feeding.

Contemporary patient cohorts acting as control data will be acquired for comparative CMR analysis. These will be derived from ethically approved local research studies from the recruiting centres for COVIDHEART using the same CMR scanners (vendor and field strength). As the patients in COVID-HEART are COVID $(+)$ and Troponin $(+)$, control populations will include:

1) A population matched for age and CVD risk factors, who have been hospitalised and $\operatorname{are} \operatorname{COVID}(+)$ and Troponin(-) [e.g. Capturing MultiORgan Effects of COVID-19 (C-MORE) cohort, Oxford, UK; NCT04510025].

2) A population matched for age and CVD risk factors, who have tested $\operatorname{COVID}(-)$ and Troponin(-).

\section{Setting}

Recruitment will take place in 25 participating secondary or tertiary care hospitals across the UK, all of whom have access to well-established local CMR services.

\section{Recruitment process and data collection}

Screening will be performed at an individual hospital level, with participating hospitals cross-referencing all admissions with a positive COVID-19 status (pathology/ radiology diagnosis), with serum troponin results. This study will integrate two key aspects. Work package-1 will establish a national image repository for patients with COVID-19 who have CMR for clinical reasons. Work package-2 (objectives as outlined above) will use CMR to characterise the nature and extent of myocardial injury in a hospitalised but recovering patient population, who are serum biomarker (troponin) positive for myocardial damage.

The source data will include hospital records, National Health Service (NHS) health and social care records, clinical and office charts, laboratory and pharmacy records as well as digital images from radiology (chest $\mathrm{X}$-ray/computed tomography/CMR) and cardiology (echocardiography/angiography/CMR). Patients in work package- 1 who have a clinically indicated CMR and are troponin positive will be invited to join work package-2 for a follow-up CMR scan and assessment at 6 months. Clinical follow up for vital status and cardiovascular events at 12 months (and up to 5-years) will be performed by recruitment sites via local hospital electronic patient records, primary care records and by national data-linkage for other NHS databases.

Co-enrolment of patients in existing registries will be encouraged (e.g. ISARIC-4c and CAPACITY: Cardiac complicAtions in Patients with SARS Corona vIrus 2 regisTrY; NCT04325412). Also, as this is a longitudinal, observational study, co-enrollment with other UK COVID-19 studies/trials will be permitted.

\section{Consent procedures}

All recruited patients will provide written informed consent as per the international ethical and scientific quality standard of Good Clinical Practice (GCP). Patient information sheets for COVID-HEART will be provided in English, and also in up to 10 other languages commonly spoken in the UK, including: French, Portuguese, Polish, Urdu, Bengali, Punjabi, Gujarati, Hindi, Somali and Arabic.

When eligibility criteria are confirmed, medical staff or appropriately trained support staff will seek consent from patients after allowing as much time as necessary to consider the study, or at least $24 \mathrm{~h}$, whilst the patient 
is either an in- or out-patient. Consent for participation in the coronary vascular sub-study (ethics reference: 19/EM/0295) will be sought at the time of consent for work package- 2 .

All participants will have the right to withdraw from the study at any point. Moreover, the investigator may discontinue a participant from the study at any time if it is considered necessary for any reason including ineligibility, significant protocol deviation or loss to follow-up. The reason for withdrawal will be recorded. If consent is withdrawn before data are used in any research analyses, then data relating to that participant could be removed, if the participant explicitly requests that their data are not used. In general, if consent is withdrawn, or if the patient were to become incapacitated, any data collected up to that point will remain on file and will be included in the final study analysis.

\section{Research investigations}

All recruited patients will be offered CMR imaging, digital 12-lead ECG, validated quality of life questionnaires, 6 min walk test (6MWT), and additional blood sampling for genetic analysis/immunological responses/T-cell function and cardiovascular biomarkers, if not already acquired. See study flow diagram (Fig. 2) and below for detailed description of research investigations.

1) CMR Scans will ideally be performed pre-discharge or within 28 days of discharge, and then repeated at 6 months ( \pm 4 weeks) from the date of admission and on the same scanner wherever possible. Scans will be performed on either a $1.5 \mathrm{~T}$ or a $3 \mathrm{~T}$ CMR system using multi-channel phased-array chest coils of any vendor, depending upon local availability. ECG gating will be employed for all cardiac gated sequences to ensure appropriate triggering. The main CMR protocol is in keeping with Society for Cardiovascular Magnetic Resonance (SCMR) recommended CMR protocols for scanning patients with active or convalescent phase COVID-19 [6], and it will take approximately 50 min to acquire, with an optional shortened and extended protocol dependent of patient preference and ability. An estimated glomerular filtration rate (eGFR) and haematocrit will be measured prior to each CMR scan. For patients with significant renal failure (eGFR $\left.<30 \mathrm{ml} / \mathrm{min} / 1.73 \mathrm{~m}^{2}\right)$, late gadolinium enhancement (LGE) and post-contrast T1-mapping can be omitted and a contrast-free CMR scan performed.

The main CMR study protocol includes the following components and typical parameters, which may vary by vendor and field strength, but remain comparable overall (Fig. 3):
A. Localiser sequences and breath-hold transverse HalfFourier Acquisition Single-shot Turbo spin Echo (HASTE) imaging stacks covering lung and abdomen to $1-2 \mathrm{~cm}$ below the kidneys. Typical sequence parameters: TE $1.33 \mathrm{~ms}$, TR $700 \mathrm{~ms}$, slice thickness $8 \mathrm{~mm}, \mathrm{FOV}=400 \mathrm{~mm}$, FOV phase 100\%, flip angle $10^{\circ}$.

B. Cine images acquired with breath-hold balanced steady-state free precession (bSSFP) sequence. Longaxis views of the LV: 4-chamber, 2-chamber, and 3-chamber views. Sequence parameters: TE $1.05 \mathrm{~ms}$, TR $40.29 \mathrm{~ms}$, slice thickness $8 \mathrm{~mm}, 25 \%$ distance factor, $\mathrm{FOV}=500 \mathrm{~mm}$, FOV phase $75 \%$, flip angle $50^{\circ}$.

C. Native (pre-contrast) T1-mapping: acquired using a single breath-hold shortened modified Look-Locker inversion (ShMOLLI) 5(1)1(1)1 technique [31], where available. Shimming will be performed to avoid artefacts. Native T1-mapping will be acquired in 3 short-axis cuts of the LV (basal, mid-ventricular, apical) to match the locations of segments 1-16 of the American Heart Association 17-segment model [32]. The apical segment 17 is omitted. Each acquisition will be verified against an $\mathrm{R}^{2}$ map (according to vendor availability). Typical pulse sequence parameters: TE $1.07 \mathrm{~ms}$, TR $379 \mathrm{~ms}$, slice thickness $8 \mathrm{~mm}$, $\mathrm{FOV}=360 \mathrm{~mm}$ (can be adjusted according to size), FOV phase $75 \%$, flip angle $35^{\circ}$, distance factor $25 \%$, generalised auto-calibrating partially parallel acquisition (GRAPPA) 2 with 24 reference lines.

D. Native (pre-contrast) T2-mapping: matching in slice location to the T1 maps, will be acquired using either a T2-prepped b-SSFP sequence with a minimum of 3 source images (e.g. MyoMaps T2-mapping for Siemens scanners), or a black-blood prepared, navigator-gated, free-breathing hybrid gradient (echo planar imaging, EPI) and spin-echo multi-echo sequence (GRASE). Typical sequence parameters: TE $1.3 \mathrm{~ms}$, TR $222.43 \mathrm{~ms}$, slice thickness $8 \mathrm{~mm}, \mathrm{FOV}=360 \mathrm{~mm}$, FOV phase $80 \%$, flip angle $20^{\circ}$.

E. Rest myocardial perfusion imaging: obtained using pixel-wise perfusion mapping (or locally available pulse sequence) following administration of $0.05 \mathrm{mmol} / \mathrm{kg}$ of gadolinium based contrast agent $(\mathrm{GBCA})$ at $4 \mathrm{ml} / \mathrm{s}$ with $20 \mathrm{ml}$ flush at $4 \mathrm{ml} / \mathrm{s}$ via a power injector. Immediately after rest perfusion image acquisition, $0.1 \mathrm{mmol} / \mathrm{kg}$ top-up (giving a total dose of $0.15 \mathrm{mmol} / \mathrm{kg}$ ) of GBCA will be given. Accepted GBCA agents include: gadobutrol and gadoteric acid. Sequence parameters: TE $1.04 \mathrm{~ms}$, TR $143.04 \mathrm{~ms}$, slice thickness $8 \mathrm{~mm}, \mathrm{FOV}=380 \mathrm{~mm}$, FOV $75 \%$, flip angle $50^{\circ}$.

F. Ventricular short-axis stack. Sequence parameters will match the cine image acquisition in long-axis. 


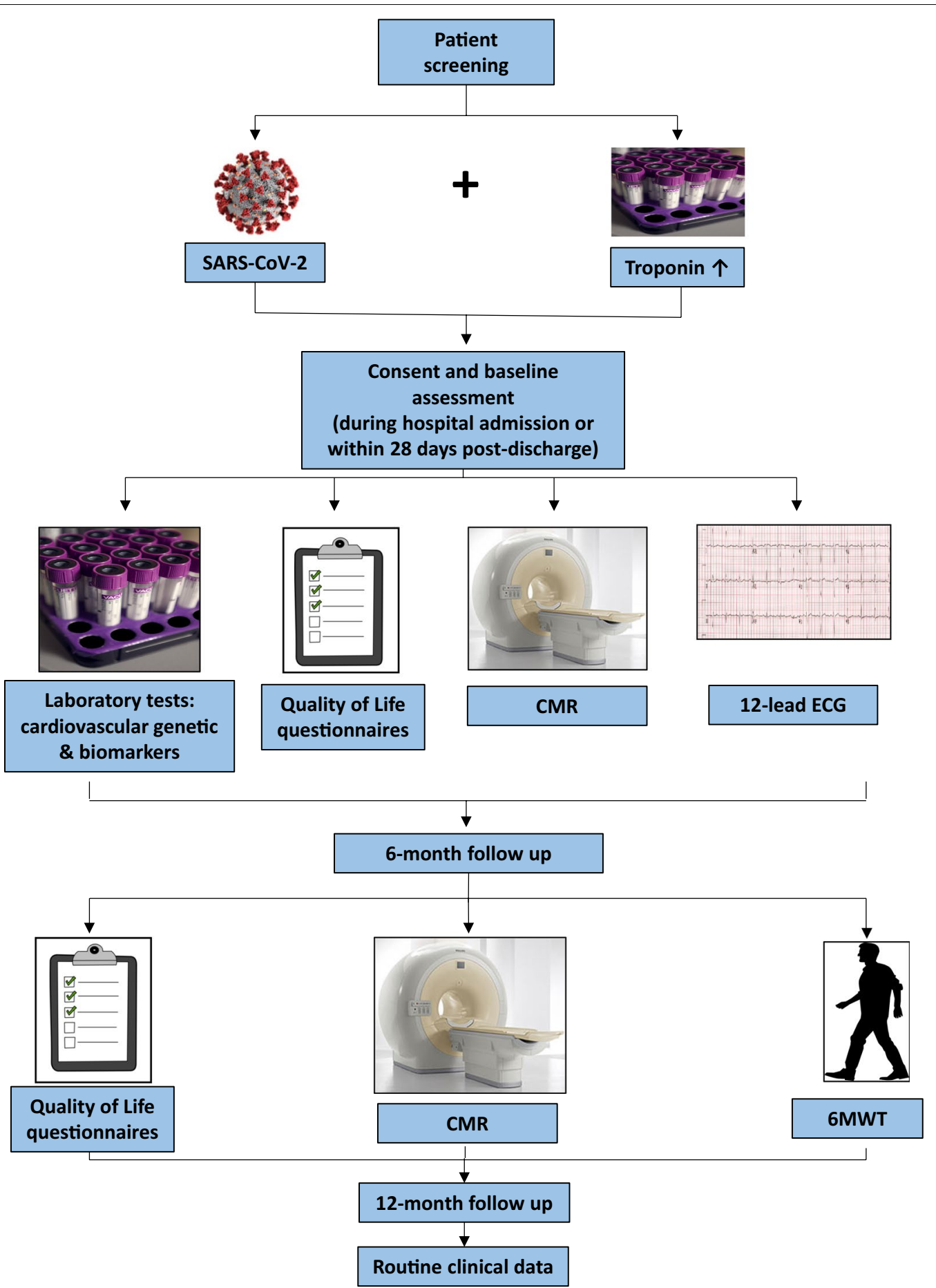

Fig. 2 Study flow diagram. COVID-19 patients, who have an elevated troponin, will be invited to participate in the study. Patients who are recruited undergo multimodality assessment at index visit: either during their hospital admission or within 28 days of discharge. Assessment includes a 12-lead ECG, CMR study, genetic and immunological blood tests, and Quality of Life questionnaires. Patients are subsequently invited for a second visit at 6 months. At this point they undergo a repeat CMR scan, quality of life assessments and 6-min walk test. Further follow up at 12 months will be conducted via examination of routine clinical data, available through general practitioners, electronic hospital records, NICOR and NHS Digital, and in collaboration with other nationally recognised COVID-19 studies 


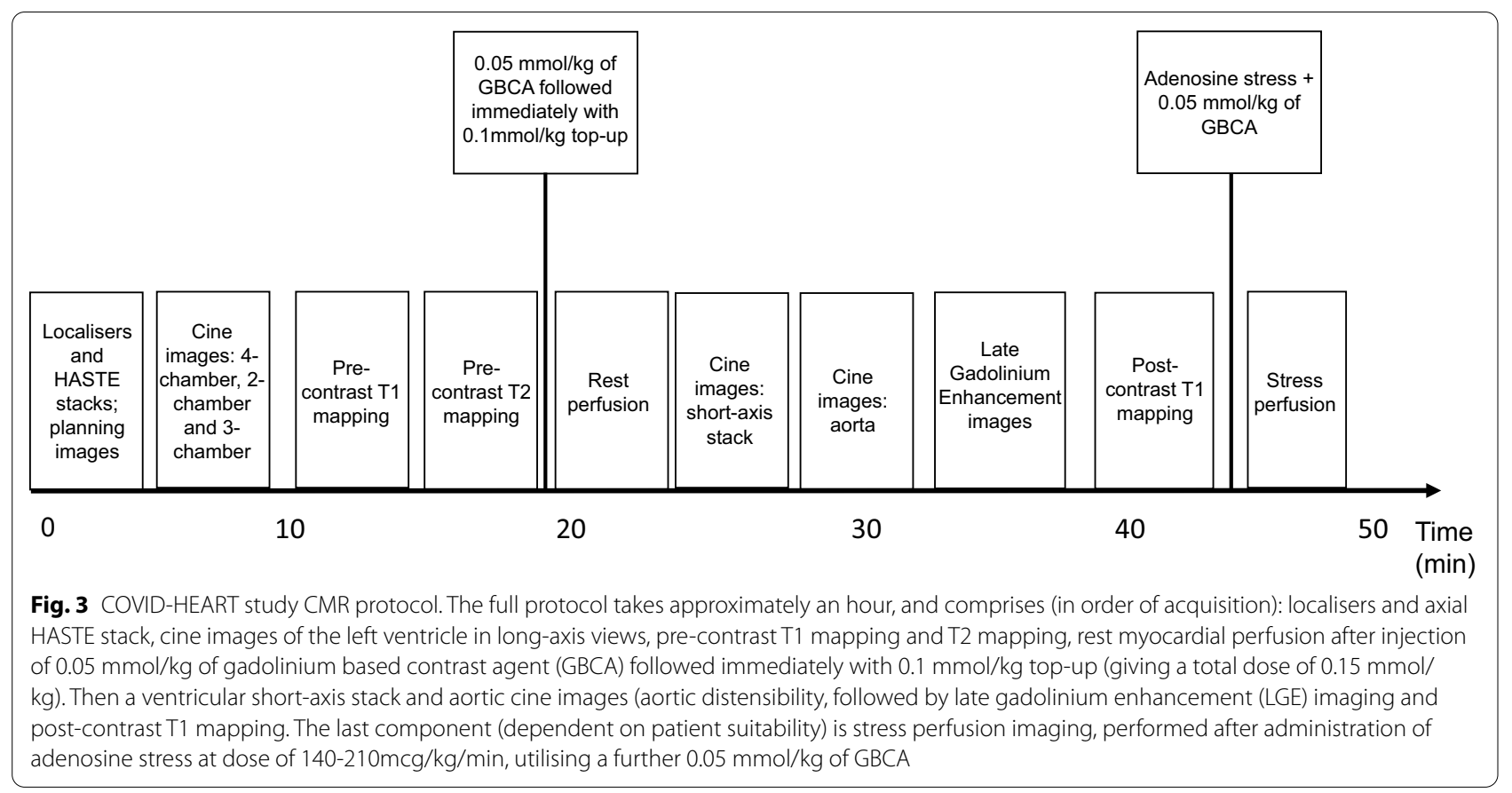

G. Aortic distensibility imaging: planned using bSSFP cine sequence of the thoracic aorta in sagittal oblique view and then acquired transverse to the ascending/descending thoracic aorta (at pulmonary artery bifurcation level). Blood pressure will be recorded whilst in the scanner to allow calculation of aortic distensibility. Sequence parameters: TE $1.25 \mathrm{~ms}$, TR $43.35 \mathrm{~ms}$, slice thickness $6 \mathrm{~mm}$, FOV $=380 \mathrm{~mm}$, FOV phase $100 \%$, flip angle $55^{\circ}, 50$ phases.

H. LGE images will be acquired $\sim 5-15$ min after intravenous injection of $0.1-0.15 \mathrm{mmol} / \mathrm{kg}$ of GBCA with a free-breathing phase-sensitive motion correction bSSFP or breath-hold, segmented inversion-recovery sequence. Contiguous stack of LV short-axis images and single long-axis slices in 2-chamber, 4-chamber and 3-chamber at the same slice locations as obtained for cine imaging will be acquired. A Look-Locker sequence will be used to determine the appropriate inversion time (TI) [33]. Example parameters are: TI will be adjusted as per Look-Locker sequence, TE $1.14 \mathrm{~ms}$, TR $411.48 \mathrm{~ms}$, matrix $128 \times 256,8 \mathrm{~mm}$ slice thickness with $2 \mathrm{~mm}$ inter-slice gap, FOV $=400 \mathrm{~mm}$, FOV phase $75 \%$, flip angle $47^{\circ}$.

I.Post-contrast $\mathrm{T} 1$ measurements will be acquired at the exact same locations as the native T1-maps and performed at least $10 \mathrm{~min}$ after injection of GBCA, using the same pulse sequence and parameters as the native T1-maps.
J. A subset of patients eligible for coronary vascular assessment will undergo an extended protocol at baseline to include stress-perfusion imaging. Adenosine stress perfusion imaging will be acquired after post-contrast T1 mapping using an adenosine dose of $140-210 \mathrm{mcg} / \mathrm{kg} / \mathrm{min}$ for 3-5 min, whilst monitoring symptoms, heart rate and blood pressure to assess an adequate response. Subsequently, GBCA will be given at a dose of $0.05 \mathrm{mmol} / \mathrm{kg}$ at $4 \mathrm{ml} / \mathrm{s}$ followed by a $20 \mathrm{ml}$ flush at $4 \mathrm{ml} / \mathrm{s}$, giving a total dose of $0.2 \mathrm{mmol} / \mathrm{kg}$. Stress perfusion imaging will be acquired in the same slice locations as the rest perfusion images. Sequence parameters will match the resting scan.

2) Digital 12-lead ECG. A 12-lead ECG will be performed either as part of routine clinical assessment or as an additional research test during the index admission and again at 6 months follow up. In selected centres (which will be provided with an identical study-specific digital ECG machine), a full 12-lead digital ECG from COVID-19 patients will be recorded daily, if possible dependent on clinical status, for up to 7 days, whilst an in-patient. Digital ECGs in patients with CMR-proven myocarditis will be compared to a reference dataset of acute ST-elevation myocardial infarction patients already available from the 
NIHR-Efficacy and Mechanism Evaluation Programme funded T-TIME randomised controlled trial [34].

3) Functional assessment by 6-min walk test. Participants will asked to perform a standard 6MWT at the 6-month visit following the American Thoracic Society guidelines [35]. Patients will be instructed to walk along a corridor and turn at $15 \mathrm{~m}$ in order to cover the maximum distance in 6 min under the supervision of study investigators. The investigators will tell the participant how much time had elapsed every 2 min and encourage participants to continue at intervals of between $30 \mathrm{~s}$ and $1 \mathrm{~min}$. At the end of 6 min, participants will be asked to stop, and the distance walked will be measured in metres.

4) Quality of life questionnaires. All participants will be asked to complete patient-reported health status questionnaires (36-Item Short Form Health Survey; SF-36v2) and a health-related QoL questionnaire (EQ-5D-5L) at baseline and 6 months. These will be performed either face to face, by telephone or by post, depending on local site and individual patient circumstances and preferences. These questionnaires were chosen to align with other UK national COVID-19 research studies (e.g. C-MORE/P-HOSP-COVID).

5) Laboratory investigations. As part of clinical routine, patients will undergo routine laboratory testing, including full blood count, renal and liver profile, inflammatory markers, and cardiovascular biomarkers (e.g. troponin and brain natriuretic peptide (BNP)). Additional research blood sampling for genetic analysis/inflammatory markers will be performed, if not already acquired from participation in other UK COVID-19 research studies. $10 \mathrm{ml}$ of blood will be drawn into an ethylenediaminetetraacetic acid (EDTA) bottle. The tube will be inverted 5 times and transferred on ice. The sample will be centrifuged for $20 \mathrm{~min}$ at $2000 \mathrm{G}$ at $4{ }^{\circ} \mathrm{C}$. The sample will be divided into $6 \times 1 \mathrm{ml}$ Thermo Matrix tubes. Subsequently a pipette will be used to provide 5 aliquots. Plasma will be taken from the centre of each sample avoiding the buffy coat layer, which will be retained separately. Samples will be labelled with a unique study ID and stored locally on-site, pending batch transfer for analysis at Leicester University and the University of Glasgow.

\section{Follow-up}

Patients will be followed up at 6 months ( \pm 4 weeks) from date of the diagnosis of COVID-19, and invited to have a second CMR scan, ECG, QoL assessment (SF-36v2 and EQ-5D-5L) and functional assessment (6MWT). In addition, routine clinical outcome data will be collated up to 5 years post-infection from: electronic patient records, general practice information systems, National Institute for Cardiovascular Outcomes Research (NICOR) and NHS Digital (eDRIS in Scotland).

\section{Study outcomes \\ Main outcomes}

1) Presence, extent, distribution of LGE

2) CMR biventricular volumes and function

3) Functional capacity (6MWT) and QoL measures

4) Long-term clinical outcomes

\section{Exploratory outcomes}

1) CMR: T1, T2 mapping indices, myocardial strain, myocardial perfusion, aortic distensibility measures.

2) ECG indices

3) Biomarker and genetic analyses

4) Coronary atheroma extent (coronary vascular substudy)

\section{Analysis plan}

The prevalence and extent of cardiac involvement in patients with COVID-19 and raised troponin will be described by CMR. Predictors of cardiac dysfunction and recovery such as: blood biomarkers, the severity of the acute infection, genetics, and comorbidities will be identified. The determinants of adverse clinical outcome in this population will also be examined. Outcome assessments (clinical event adjudication) will be undertaken at individual hospital sites according a pre-specified standard definition set (Appendix 1) contained with a signed clinical outcome charter.

1) CMR data: All quantitative analyses will be performed blinded to participant status. CMR data sets will be analysed by a disseminated core-lab technique as previously used in the BSCMR UK valve consortium [36]. LV structure and function including mass and wall thickness will be analyzed using a clinically validated artificial intelligence (AI) analysis platform [37]. Left atrial area and global longitudinal shortening will be similarly analyzed by further validated artificial intelligence approaches [38]. LGE patterns will also be classified into patterns reflecting likely aetiology and quantitative assessment will be performed by semi-automated signal intensity analysis according to the full width at half maximum technique and other thresholds where appropriate [36]. Evidence of pericarditis will be assessed on cine, LGE and tissue characterisation images. T1 and T2 mapping will be quality-assured and analysed to establish the 
global and segmental T1 and T2 values, using standardised approaches developed by the Oxford group [39, 40]. Myocardial perfusion assessment for the coronary vascular sub-study will be carried out by segmental and global quantitative myocardial blood flow calculation during rest and adenosine stress; it will be calculated from fully automated in-line quantification [41, 42]. In addition, visual perfusion defects will be scored semiquantitatively by 2 observers as previously described [43] with disagreement resolved by a third reader. The likelihood of microvascular disease (patchy perfusion defects not following the distribution of a coronary distribution or circumferential defects not affecting apical segments) will be recorded. Circumferential strain and strain rates will be generated by automated tissue tracking software (cvi42, Circle Cardiovascular Imaging, Alberta, Calgary, Canada) as previously described [44]. Aortic distensibility will be assessed using blood pressure assessments and measurements of the cross-sectional area of the ascending aorta in systole and diastole on axial cine slices at the level of the right pulmonary artery. Distensibility $=($ aortic max lumen area - aortic min lumen area)/(aortic min lumen area * [systolic blood pressure-diastolic blood pressure]).

2) ECG data: The standard and digital 12-lead ECGs will be electronically transferred via the study portal into the image repository at the coordinating centre in Glasgow, Scotland. Digital ECGs will be analysed in the University of Glasgow ECG core lab utilising the previously validated Glasgow ECG analysis software [45]. A full set of measurements plus interpretation will be extracted for analysis of ECG changes and statistical processing, with the aim of determining sensitive and specific criteria for diagnosing myocarditis from the ECG, and prognostic ECG findings in this population [46]. ECGs available in printed form will also be electronically transmitted in pdf format to the ECG Core Lab for standardised review based on the same criteria as used in the Glasgow program. Manually classified changes will also be subject to statistical analysis.

3) Laboratory data: All blood samples will be transferred to the central coordinating centre in Leicester, England and analysed in the University of Leicester Cardiovascular Research Centre and the BHF Glasgow Cardiovascular Research Centre, University of Glasgow. Analysis will entail novel cardiovascular biomarkers (e.g. vascular cell adhesion molecule, interleukin-6) and markers of genetic susceptibility to COVID-19. The genetic analysis will be used to explore links specifically in the black and minority ethnic populations and linked in with existing cohorts for genotyping (The Genetics and Vascular Health Check study, The Biomedical Research Informatics Centre for Cardiovascular Science and UK Biobank).

4) 6MWT: As per the American Thoracic Society guidelines, the distance walked (measured in meters) in $6 \mathrm{~min}$ will be recorded by the investigator supervising the test. Symptoms of perceived breathlessness will be recorded on the Borg scale and if the test was stopped early, the reason will be recorded.

5) Quality of life questionnaires: Patients' responses to the 36-Item Short Form Health Survey and the health-related QoL questionnaire (EQ-5D-5L) will be recorded in the eCRF by the investigators. Each of the 8 health domains measured by the SF-36v2 will be given a combined percentage score, with higher percentage scores representing a better level of functioning. Each of the 5 domains of the EQ-5D-5L will be scored on a 1-5 scale, with level 1 indicating no problems with a particular domain and level 5 indicating extreme problems. Health state of an individual patient will be determined by combining the levels from all the domains.

\section{Statistical considerations Sample size considerations}

Patients in work package- 1 will originate from the open national image repository for all UK CMR centres, and therefore no sample size calculation is required. For work package-2, based on the assumption that the mean prevalence of myocardial involvement was $12 \%$ (midrange) from previous studies, a precision of $3.5 \%$ and $95 \%$ confidence level, a sample size of $333 \pm 3.5 \%$ would be required. To account for a $10 \%$ drop out rate, it was calculated that 370 patients are needed.

\section{Statistical analysis}

Statistical analyses will be conducted after production of a signed statistical analysis plan and once baseline and follow-up data collection has been completed. Broadly, the statistical methods for each study objective will be as follows:

1) to characterise the nature and extent of myocardial involvement in COVID-19 patients with an elevated troponin, analyses will be descriptive, and will involve estimation of prevalence with $95 \%$ confidence intervals (CIs);

2) to assess how cardiac involvement and clinical outcomes associate with recognised risk factors for mortality in COVID-19 (age, sex, ethnicity and comorbidities) and with genetic factors, we shall present tabular and graphical descriptive summaries within 
subgroups, and use logistic regression to test for associations and provide estimates with 95\% CIs;

$3)$ to evaluate if differences in myocardial recovery at 6 months are associated with demographics, genetics and $\mathrm{CV}$ comorbidities (diabetes, hypertension, heart failure and $\mathrm{CAD} /$ peripheral vascular disease), descriptive summaries, and linear and logistic regression methods will be applied;

4) to understand the association between recovery status at 6 months and patient-reported QoL and functional capacity, descriptive methods, and linear regression will be used.

To assess whether there are characteristic ECG findings specific for SARS-CoV-2 myocarditis, measures of diagnostic performance will be calculated, and ROC analyses will be used.

In the coronary vascular sub-study, descriptive methods will be used to establish the nature and prevalence of myocardial ischaemia and microvascular dysfunction, and linear regression methods, including mixed effects methods for modelling myocardial blood flow within segments 1-16 of the American Heart Association 17-segment model, will be used to assess risk factors.

Throughout these analyses, levels of missing data will be reported, and regression models will be fitted using multiple imputation of missing predictor variables. Where appropriate, data from other cohorts will be used as control populations.

\section{Data monitoring and linkage}

The University of Glasgow will be coordinating centre for data and analysis and will hold identifiable data in secured databases to permit record linkage. The Universities of Leicester and Glasgow and NHS Laboratory Medicine (including the Biorepository) will undertake storage and/or analysis of blood samples (DNA, RNA, and small molecules).

As part of the patient consent process, it will be explicitly requested that anonymised data and images can be shared with other national and global research initiatives, for efficiency in data collection and sharing across other nationally prioritised UK COVID studies (e.g. C-MORE, P-HOSP-COVID and CISCO-19 [Cardiovascular and Pulmonary Imaging in SARS Coronavirus disease-19]) [47], as well as with other international CMR studies and registries e.g. the international SCMR COVID-19 registry. The principles set out in the 2016 Statement on data sharing in public health emergencies will be followed and it will be ensured that the World Health Organization (WHO) has rapid access to the emerging findings that could aid the global response.
As this is an observational study only serious adverse events (SAEs) that relate directly to the participation in the study procedures and sample collection will be reported to the sponsor and Ethics committee. All hospitalisations and deaths will be recorded as study outcomes.

The management of incidental findings of the research CMR scans will be performed at the individual site level according to the normal local practice and procedures.

\section{Discussion}

The COVID-HEART study is a longitudinal, multicentre, observational cohort study of UK patients presenting with COVID-19 infection and positive troponin, who are either recovering in hospital or were recently discharged. It aims to utilise CMR in evaluation of the nature and extent of myocardial injury and subsequently, myocardial recovery in these patients, and to examine the impact of myocardial recovery status on quality of life and functional capacity. Additional investigations include laboratory testing, which will encompass routine tests, novel cardiac biomarkers, and genetic analysis. These results will be shared with other global initiatives to address genetic susceptibility to COVID-19. Furthermore, ECGs will be investigated with the aim of identifying sensitive and specific changes that would allow diagnosis of myocarditis without the need for invasive testing.

As COVID-19 is an unprecedented global health emergency and previous studies showed that a significant proportion of patients had myocardial injury, it is important to further understand the underlying pathophysiology, risk factors and long-term outcomes. Therefore, the results of the COVID-HEART study, and the results of other registries examining myocardial injury (e.g. PHOSP-COVID, ISRCTN10980107; MOIST, NCT04525404; MYOCOVID, NCT04375748; MIIC-MI, NCT04412369; CARDOVID, NCT04455347; CISCO19, NCT04403607), may have significant clinical implications on the assessment and management of these patients. Furthermore, the new knowledge from these studies will help inform disease-targeted therapy development, for the prevention and treatment of cardiovascular complications in COVID-19.

\section{Appendix 1: Summary definitions of clinical outcomes}

A pre-specified, signed, Clinical Outcomes Charter will be provided to each recruitment centre. This will contain detailed endpoint definitions to facilitate consistent local adjudication and unbiased reporting. Endpoint definitions will align with the 2014 American College of Cardiology/American Heart Association Key Data Elements 
and Definitions for Cardiovascular Endpoint Events in Clinical Trials [48]. In brief, these endpoints will include:

1) Cardiovascular death is a defined as death occurring secondary to an acute myocardial infarction, sudden cardiac death, heart failure, stroke, death resulting from cardiovascular procedures or death due to cardiovascular haemorrhage.

2) Non-cardiovascular death is defined as any death with a specific cause that is not thought to be due to a cardiovascular cause. There should be unequivocal and documented evidence of a non-cardiovascular cause of death.

3) Myocardial infarction is defined as elevation of cardiac biomarkers above the 99th percentile of the upper reference limit in the setting of an appropriate clinical presentation in combination with diagnostic ischaemic electrocardiographic changes. Specific clinical classification of different types of myocardial infarction will be assigned from the 4th Universal Definition of Myocardial Infarction [12].

4) Coronary artery revascularisation, either by percutaneous coronary intervention (PCI) or coronary artery bypass graft surgery (CABG).

5) Transient ischaemic attack is defined as an episode of focal neurological dysfunction resulting from brain, retinal and spinal cord ischaemia, that is transient in nature and resolves within $24 \mathrm{~h}$.

6) Stroke is defined as a focal or global neurological dysfunction secondary to brain, retinal or spinal cord injury resulting from haemorrhage or infarction.

7) Pulmonary embolism is defined based upon clinical evaluation and a diagnostic imaging test, ideally, computed tomography (CT) pulmonary angiography. If ventilation/perfusion scintigraphy is used, then the diagnosis may be accepted based on clinical evaluation and a high probability scan. The clinical evaluation and diagnosis of pulmonary embolism should align with the 2019 ESC Guidelines for the diagnosis and management of acute pulmonary embolism [49].

8) Deep venous thrombosis (DVT) diagnosis should be established based on a clinical evaluation, D-dimer elevation and imaging findings (duplex ultrasound or CT angiography).

9) Myopericarditis diagnosis will be adjudicated based upon the 2013 Position Statement of the European Society of Cardiology Working Group on Myocardial and Pericardial Diseases.(50)

10) Hospitalisation for other cardiovascular cause:

a) Heart failure events are defined as hospitalisation for heart failure or an urgent outpatient visit. A heart failure hospitalisation event must fulfil the following criteria: primary admission diagnosis is heart failure, the patient experiences either worsening of heart failure symptoms or a new symptom, and requires an increase in therapy.

b) Arrhythmia defined as hospitalisation with an arrhythmia (atrial, supraventricular and/or ventricular or brady-arrhythmia) documented by electrocardiography (single- or multi-lead) with a change in treatment (withhold, change in dose of existing medication, prescription of new medication for arrhythmia) and/or pacemaker therapy.

c) Chest pain/unstable angina defined as an emergency/unplanned admission to a hospital setting that results in at least one overnight stay (i.e. a date change) with cardiac ischaemic-type symptoms at rest or minimal exertion.

\section{Abbreviations}

6MWT: Six minute walk test; BNP: Brain natriuretic peptide; BSCMR: British Society of Cardiovascular Magnetic Resonance; bSSFP: Balanced steady state free precession; C-MORE: Capturing Multi-ORgan Effects of COVID-19; CAD: Coronary artery disease; CMR: Cardiovascular magnetic resonance; COVID-19: Coronavirus disease 2019; CVD: Cardiovascular disease; ECG: Electrocardiogram; eCRF: Electronic case report form; ECV: Extracellular volume fraction; EDTA: Ethylenediaminetetraacetic acid; eGFR: Estimated glomerular filtration rate; EPI: Echo planar imaging; FBC: Full blood count; FOV: Field-of-view; GBCA: Gadolinium-based contrast agent; GCP: Good clinical practice; GRAPPA: Generalised auto-calibrating partially parallel acquisition; GRE: Gradient recalled echo; HASTE: Half-Fourier acquisition single-shot turbo spin echo; LGE: Late gadolinium enhancement; LV: Left ventricle/left ventricular; LVOT: Left ventricular outflow tract; NHS: National Health Service; NICOR: The National Institute for Cardiovascular Outcomes Research; P-HOSP-COVID: Post-hospitalisation COVID-19 study; QoL: Quality of life; RNA: Ribonucleic acid; RV: Right ventricle/ right ventricular; SAEs: Serious adverse events; SARS-CoV-2: Severe acute respiratory syndrome coronavirus 2; SCMR: Society for Cardiovascular Magnetic Resonance; SF-36: 36-Item Short Form Health Survey; ShMOLLI: Shortened Modified Look-Locker Inversion recovery; TE: Echo time; TI: Inversion time; TR: Repetition time; NIHR-UKRI: National Institute for Health Research—UK Research Institute; VLA: Vertical long axis; WHO: World Health Organization.

\section{Acknowledgements}

CB acknowledges British Heart Foundation support (RE/18/6134217). GPM is funded by a NIHR Research Professorship (RP-2017-08-ST2-007). CM is funded by a NIHR Clinician Scientist Award (CS-2015-15-003). VMF and SN acknowledge the NIHR Oxford BRC for support of this study. CBD is in part supported by the NIHR Biomedical Research Centre at University Hospitals Bristol NHS Foundation Trust and the University of Bristol. Additional support was provided by the NIHR Leicester Biomedical Research Centre and the NIHR Leeds Clinical Research Facility. The views expressed in this publication are those of the author(s) and not necessarily those of the NHS, the National Institute for Health Research or the Department of Health and Social Care. We thank the patients and staff who have supported this project. Dr. Warren J. Manning served as a Guest Editor for this manuscript. Study Management and Recruitment centres: Grant applicants: JP Greenwood (chief investigator), GP McCann, C Berry, M Dweck, J Moon, CM Miller, A Chiribiri, S Prasad, VM Ferreira, C Bucciarelli-Ducci, D Dawson. Data repository and statistical analysis: Glasgow Clinical Trials Unit. Senior study statistician: Prof A McConnachie, GCTU. Local Principle Investigators and Recruitment Centres: Prof John Greenwood, Leeds Teaching Hospitals NHS Trust, UK; Prof Gerry McCann, Glenfield Hospital, Leicester, UK; Prof Dana Dawson, Aberdeen Royal Infirmary, UK; Prof Marc Dweck, Royal Infirmary of Edinburgh, UK; Prof Vanessa Ferreira, John 
Radcliffe Hospital, Oxford, UK; Prof Colin Berry, Queen Elizabeth University Hospital, Glasgow, UK; Dr Peter Swoboda, Pinderfields Hospital, Wakefield, UK; Dr Richard Steeds, Queen Elizabeth Hospital, Birmingham, UK; Prof James Moon, UCL Hospital London, UK; Dr Christopher Miller, Wythenshawe Hospital, Manchester, UK; Dr Timothy Fairbairn, Liverpool Heart and Chest Hospital, UK; Dr Andrew Flett, Southampton General Hospital, UK; Prof Marianna Fontana, Royal Free Hospital, London, UK; Dr Thomas Green, Northumbria NHS Trust, UK; Prof Amedeo Chiribiri, St Thomas' Hospital, London, UK; Dr Chiara Bucciarelli-Ducci, University Hospitals Bristol and Weston NHS Trust, UK; Dr Graham Cole, Hammersmith Hospital, London, UK; Prof Sanjay Prasad, Royal Brompton Hospital, London, UK; Dr Adam McDiarmid, Freeman Hospital, Newcastle Upon Tyne, UK; Dr Nicholas Bunce, St Georges Hospital, London, UK; Dr Prathap Kanagala, Aintree University Hospital, Liverpool, UK; Prof Nicholas Bellenger, The Royal Devon and Exeter Hospital, UK; Dr Tishi Ninan, Swansea Bay University Hospital, UK; Dr Khaled Alfakih, Lewisham University Hospital, London, UK; Prof James Moon, St Bartholomew's Hospital, London, UK.

\section{Authors' contributions}

All authors have participated in the design of the study and helped to draft the manuscript. All authors read and approved the final manuscript.

\section{Funding}

COVID-HEART is funded by the National Institute for Health Research (NIHR) and UK Research and Innovation (UKRI) COVID-19 Rapid Response Rolling Call (Grant Number COV0254), and sponsored by the University of Leeds, UK. The study has been endorsed by the British Society of Cardiovascular Magnetic Resonance (BSCMR) Research Group, and nationally prioritised, and received both BHF-NIHR Cardiovascular Partnership Flagship Status, and the NIHR Urgent Public Health Group identified it as an Urgent Public Health (UPH) study. Funding for the translation of the patient information leaflets into nonEnglish languages was provided by the West Yorkshire and Humber Clinical Research Network (CV070).

\section{Availability of data and materials}

The datasets used and/or analysed during the current study will be available from the corresponding author on reasonable request. Anonymised data may be shared with other COVID-19 global initiatives.

\section{Declarations}

\section{Ethics approval and consent for participation}

Granted by the North West-Greater Manchester South Research Ethics Committee (20/NW/0292). All patients provided written informed consent.

\section{Consent for publication}

All patients have provided informed written consent. The funder and sponsor had no involvement in either the decision to publish, or, the published content.

\section{Competing interests}

$\mathrm{CB}$ is employed by the University of Glasgow which holds research and/or consultancy agreements with AstraZeneca, Abbott Vascular, Boehringer Ingelheim, GSK, HeartFlow, Opsens, and Novartis. CBD is the chief executive officer (part-time) of the Society for Cardiovascular Magnetic Resonance (SCMR). All other authors have no conflicts to declare.

\section{Author details}

${ }^{1}$ Institute of Cardiovascular and Metabolic Medicine, University of Leeds, Leeds LS2 9JT, UK. ${ }^{2}$ University of Leicester and The NIHR Leicester Biomedical Research Centre, Glenfield Hospital, Leicester, UK. ${ }^{3}$ Institute of Cardiovascular and Medical Sciences and British Heart Foundation Glasgow Cardiovascular Research Centre, University of Glasgow, Glasgow, UK. ${ }^{4}$ Division of Cardiovascular Medicine, Radcliffe Department of Medicine, Oxford Centre for Clinical Magnetic Resonance Research, British Heart Foundation Centre of Research Excellence, Oxford NIHR Biomedical Research Centre, University of Oxford, Oxford, UK. ${ }^{5}$ Institute of Cardiovascular Science, University College London, London, UK. ' Division of Cardiovascular Sciences, School of Medical Sciences, Faculty of Biology, Medicine and Health, University of Manchester, Manchester, UK. ${ }^{7}$ School of Biomedical Engineering and Imaging Sciences, King's College London, BHF Centre of Excellence and the NIHR Biomedical Research Centre at Guy's and St. Thomas' NHS Foundation Trust, The Rayne Institute, St. Thomas' Hospital, London, UK. ${ }^{8}$ National Heart and Lung Institute, Imperial College, London, UK. ${ }^{9}$ University of Edinburgh and British Heart Foundation Centre for Cardiovascular Science, Edinburgh, UK. ${ }^{10}$ Bristol Heart Institute, Bristol NIHR Cardiovascular Research Centre, University of Bristol and University Hospitals Bristol and Weston NHS Trust, Bristol, UK. ${ }^{11}$ Department of Cardiology, Aberdeen Cardiovascular and Diabetes Centre, Aberdeen Royal Infirmary and University of Aberdeen, Aberdeen, UK. ${ }^{12}$ Division of Medicine, Royal Free Hospital, University College London, London, UK. ${ }^{13}$ Electrocardiology Core Laboratory, Institute of Health and Wellbeing, University of Glasgow, Glasgow, UK. ${ }^{14}$ Robertson Centre for Biostatistics, Institute of Health and Wellbeing, University of Glasgow, Glasgow, UK.

\section{Received: 8 February 2021 Accepted: 2 April 2021}

Published online: 10 June 2021

\section{References}

1. Bandyopadhyay D, Akhtar T, Hajra A, Gupta M, Das A, Chakraborty S, et al. COVID-19 pandemic: cardiovascular complications and future implications. Am J Cardiovasc Drugs. 2020;20(4):311-24.

2. Gavriatopoulou M, Korompoki E, Fotiou D, Ntanasis-Stathopoulos I, Psaltopoulou T, Kastritis E, et al. Organ-specific manifestations of COVID-19 infection. Clin Exp Med. 2020. https://doi.org/10.1007/ s10238-020-00648-x.

3. Raman B, Cassar MP, Tunnicliffe EM, Filippini N, Griffanti L, Alfaro-Almagro $F$, et al. Medium-term effects of SARS-CoV-2 infection on multiple vital organs, exercise capacity, cognition, quality of life and mental health, post-hospital discharge. EClinicalMedicine. 2020;31:100683.

4. Bansal M. Cardiovascular disease and COVID-19. Diabetes Metab Syndr. 2020;14(3):247-50.

5. Aghagoli G, Gallo Marin B, Soliman LB, Sellke FW. Cardiac involvement in COVID-19 patients: risk factors, predictors, and complications: a review. J Cardiac Surg. 2020. https://doi.org/10.1111/jocs.14538.

6. Kelle S, Bucciarelli-Ducci C, Judd RM, Kwong RY, Simonetti O, Plein S, et al. Society for Cardiovascular Magnetic Resonance (SCMR) recommended CMR protocols for scanning patients with active or convalescent phase COVID-19 infection. J Cardiovasc Magn Reson. 2020;22(1):61.

7. Clerkin Kevin J, Fried Justin A, Raikhelkar J, Sayer G, Griffin Jan M, Masoumi A, et al. COVID-19 and cardiovascular disease. Circulation. 2020;141(20):1648-55.

8. Giustino G, Croft Lori B, Stefanini Giulio G, Bragato R, Silbiger Jeffrey J, Vicenzi $\mathrm{M}$, et al. Characterization of myocardial injury in patients with COVID-19. J Am Coll Cardiol. 2020;76(18):2043-55.

9. Puntmann VO, Carerj ML, Wieters I, Fahim M, Arendt C, Hoffmann J, et al. Outcomes of cardiovascular magnetic resonance imaging in patients recently recovered from coronavirus disease 2019 COVID-19. JAMA Cardiol. 2020. https://doi.org/10.1001/jamacardio.2020.3557.

10. Libby P. The heart in COVID-19. JACC Basic TransI Sci. 2020;5(5):537-42.

11. Song Y, Gao P, Ran T, Qian H, Guo F, Chang L, et al. High Inflammatory burden: a potential cause of myocardial injury in critically ill patients with COVID-19. Front Cardiovasc Med. 2020;7:128

12. Thygesen K, Alpert JS, Jaffe AS, Chaitman BR, Bax JJ, Morrow DA, et al. Fourth universal definition of myocardial infarction (2018). Eur Heart J. 2019;40(3):237-69.

13. Klok FA, Kruip M, Van Der Meer N, Arbous M, Gommers D, Kant K, et al. Confirmation of the high cumulative incidence of thrombotic complications in critically ill ICU patients with COVID-19: an updated analysis. Thromb Res. 2020. https://doi.org/10.1016/j.thromres.2020.04.041.

14. Pellegrini D, Kawakami R, Guagliumi G, Sakamoto A, Kawai K, Gianatti A, et al. Microthrombi as a major cause of cardiac injury in COVID-19: a pathologic study. Circulation. 2021. https://doi.org/10.1161/CIRCULATIO NAHA.120.051828.

15. Stefanini GG, Montorfano M, Trabattoni D, Andreini D, Ferrante G, Ancona $M$, et al. ST-elevation myocardial infarction in patients with COVID-19: clinical and angiographic outcomes. Circulation. 2020;141(25):2113-6.

16. Cosyns B, Lochy S, Luchian ML, Gimelli A, Pontone G, Allard SD, et al. The role of cardiovascular imaging for myocardial injury in hospitalized COVID-19 patients. Eur Heart J Cardiovasc Imaging. 2020;21 (7):709-14. 
17. Karbalai Saleh S, Oraii A, Soleimani A, Hadadi A, Shajari Z, Montazeri M, et al. The association between cardiac injury and outcomes in hospitalized patients with COVID-19. Intern Emerg Med. 2020;15(8):1415-24.

18. Nie S-F, Yu M, Xie T, Yang F, Wang H-B, Wang Z-H, et al. Cardiac Troponin $\mathrm{I}$ is an independent predictor for mortality in hospitalized patients with COVID-19. Circulation. 2020;142(6):608-10

19. Mitrani RD, Dabas N, Goldberger JJ. COVID-19 cardiac injury: Implications for long-term surveillance and outcomes in survivors. Heart Rhythm. 2020;17(11):1984-90.

20. Lala A, Johnson Kipp W, Januzzi James L, Russak Adam J, Paranjpe I, Richter F, et al. Prevalence and impact of myocardial injury in patients hospitalized with COVID-19 infection. J Am Coll Cardiol. 2020;76(5):533-46.

21. Zhou F, Yu T, Du R, Fan G, Liu Y, Liu Z, et al. Clinical course and risk factors for mortality of adult inpatients with COVID-19 in Wuhan, China: a retrospective cohort study. Lancet. 2020. https://doi.org/10.1016/S01406736(20)30566-3.

22. Li B, Yang J, Zhao F, Zhi L, Wang X, Liu L, et al. Prevalence and impact of cardiovascular metabolic diseases on COVID-19 in China. Clin Res Cardiol. 2020;109(5):531-8.

23. Novel CPERE. The epidemiological characteristics of an outbreak of 2019 novel coronavirus diseases (COVID-19) in China. Zhonghua Liu Xing Bing Xue Za Zhi. 2020;41(2):145.

24. Sandoval $Y$, Januzzi JL, Jaffe AS. Cardiac troponin for assessment of myocardial injury in COVID-19: JACC review topic of the week. J Am Coll Cardiol. 2020;76(10):1244-58.

25. Emrich T, Emrich K, Abegunewardene N, Oberholzer K, Dueber C, Muenzel T, et al. Cardiac MR enables diagnosis in $90 \%$ of patients with acute chest pain, elevated biomarkers and unobstructed coronary arteries. Br J Radiol. 2015:88(1049):20150025.

26. Ponikowski P, Voors AA, Anker SD, Bueno H, Cleland JG, Coats AJ, et al. 2016 ESC Guidelines for the diagnosis and treatment of acute and chronic heart failure: The Task Force for the diagnosis and treatment of acute and chronic heart failure of the European Society of Cardiology (ESC) developed with the special contribution of the Heart Failure Association (HFA) of the ESC. Eur Heart J. 2016;37(27):2129-200.

27. Friedrich MG, Sechtem U, Schulz-Menger J, Holmvang G, Alakija P, Cooper $\mathrm{LT}$, et al. Cardiovascular magnetic resonance in myocarditis: a JACC White Paper. J Am Coll Cardiol. 2009;53(17):1475-87.

28. Ferreira VM, Schulz-Menger J, Holmvang G, Kramer CM, Carbone I, Sechtem U, et al. Cardiovascular magnetic resonance in nonischemic myocardial inflammation: expert recommendations. J Am Coll Cardiol. 2018:72(24):3158-76

29. Huang L, Zhao P, Tang D, Zhu T, Han R, Zhan C, et al. Cardiac involvement in patients recovered from COVID-2019 identified using magnetic resonance imaging. JACC Cardiovasc Imaging. 2020;13(11):2330-9.

30. Plein S, Kozerke S, Suerder D, Luescher TF, Greenwood JP, Boesiger P, et al. High spatial resolution myocardial perfusion cardiac magnetic resonance for the detection of coronary artery disease. Eur Heart J. 2008:29(17):2148-55.

31. Piechnik S, Ferreira VM, Dall'Armellina E, Cochlin LE, Greiser A, Neubauer S, Robson MD. Shortened Modified Look-Locker Inversion recovery (ShMOLLI) for clinical myocardial T1-mapping at 1.5 and 3 T within a 9 heartbeat breathhold. J Cardiovasc Magn Reson. 2010;12:69.

32. Cerqueira MD. American Heart Association Writing Group on Myocardial Segmentation and Registration for Cardiac Imaging: standardized myocardial segmentation and nomenclature for tomographic imaging of the heart: a statement for healthcare professionals from the Cardiac Imaging Committee of the Council on Clinical Cardiology of the American Heart Association. Circulation. 2002;105:539-42.

33. Look DC, Locker DR. Time saving in measurement of NMR and EPR relaxation times. Rev Sci Instrum. 1970;41(2):250-1.

34. Maznyczka AM, McCartney PJ, Eteiba H, Greenwood JP, Muir DF, Chowdhary S, et al. One-year outcomes after low-dose intracoronary alteplase during primary percutaneous coronary intervention: the T-TIME randomized trial. Circ Cardiovasc Interv. 2020;13(2):e008855.

35. American TS. ATS statement: guidelines for the six-minute walk test. Am J Respir Crit Care Med. 2002;166:111-7.
36. Musa TA, Treibel TA, Vassiliou VS, Captur G, Singh A, Chin C, et al. Myocardial scar and mortality in severe aortic stenosis: data from the BSCMR valve consortium. Circulation. 2018;138(18):1935-47.

37. Augusto JB, Davies RH, Bhuva AN, Knott KD, Seraphim A, Alfarih M, et al. Diagnosis and risk stratification in hypertrophic cardiomyopathy using machine learning wall thickness measurement: a comparison with human test-retest performance. Lancet Digit Health. 2020;3(1):e20-8.

38. Maceira AM, Cosín-Sales J, Roughton M, Prasad SK, Pennell DJ. Reference left atrial dimensions and volumes by steady state free precession cardiovascular magnetic resonance. J Cardiovasc Magn Reson. 2010;12(1):1-10.

39. Carapella V, Puchta H, Lukaschuk E, Marini C, Werys K, Neubauer S, et al. Standardized image post-processing of cardiovascular magnetic resonance T1-mapping reduces variability and improves accuracy and consistency in myocardial tissue characterization. Int J Cardiol. 2020;298:128-34

40. Zhang Q, Werys K, Popescu IA, Biasiolli L, Ntusi NAB, Desai M, et al. Quality assurance of quantitative cardiac T1-mapping in multicenter clinical trials - a T1 phantom program from the hypertrophic cardiomyopathy registry (HCMR) study. Int J Cardiol. 2021. https://doi.org/10.1016/j.jjcard. 2021.01.026.

41. Kellman P, Hansen MS, Nielles-Vallespin S, Nickander J, Themudo R, Ugander $M$, et al. Myocardial perfusion cardiovascular magnetic resonance: optimized dual sequence and reconstruction for quantification. J Cardiovasc Magn Reson. 2017;19(1):1-14.

42. Knott KD, Seraphim A, Augusto JB, Xue H, Chacko L, Aung N, et al. The prognostic significance of quantitative myocardial perfusion: an artificial intelligence-based approach using perfusion mapping. Circulation. 2020;141(16):1282-91.

43. Hussain ST, Paul M, Plein S, McCann GP, Shah AM, Marber MS, et al. Design and rationale of the MR-INFORM study: stress perfusion cardiovascular magnetic resonance imaging to guide the management of patients with stable coronary artery disease. J Cardiovasc Magn Reson. 2012;14(1):1-10.

44. Graham-Brown M, Gulsin G, Parke K, Wormleighton J, Lai F, Athithan $L$, et al. A comparison of the reproducibility of two cine-derived strain software programmes in disease states. Eur J Radiol. 2019:113:51-8.

45. Macfarlane PW, Devine B, Clark E. The University of Glasgow (Uni-G) ECG analysis program. Comput Cardiol. 2005;32:451-4.

46. Fischer K, Marggraf M, Stark AW, Kaneko K, Aghayev A, Guensch DP, et al. Association of ECG parameters with late gadolinium enhancement and outcome in patients with clinical suspicion of acute or subacute myocarditis referred for CMR imaging. PLoS ONE. 2020;15(1):e0227134.

47. Mangion K, Morrow A, Bagot C, Bayes H, Blyth KG, Church C, et al. The chief scientist office cardiovascular and pulmonary imaging in SARS Coronavirus disease-19 (CISCO-19) study. Cardiovasc Res. 2020;116(14):2185-96.

48. Hicks KA, Mahaffey KW, Mehran R, Nissen SE, Wiviott SD, Dunn B, et al. 2017 Cardiovascular and stroke endpoint definitions for clinical trials. Circulation. 2018;137(9):961-72.

49. Konstantinides SV, Meyer G, Becattini C, Bueno H, Geersing G-J, Harjola $V$-P, et al. 2019 ESC Guidelines for the diagnosis and management of acute pulmonary embolism developed in collaboration with the European Respiratory Society (ERS) The Task Force for the diagnosis and management of acute pulmonary embolism of the European Society of Cardiology (ESC). Eur Heart J. 2020;41 (4):543-603.

50. Caforio AL, Pankuweit S, Arbustini E, Basso C, Gimeno-Blanes J, Felix SB, et al. Current state of knowledge on aetiology, diagnosis, management, and therapy of myocarditis: a position statement of the European Society of Cardiology Working Group on Myocardial and Pericardial Diseases. Eur Heart J. 2013;34(33):2636-48.

\section{Publisher's Note}

Springer Nature remains neutral with regard to jurisdictional claims in published maps and institutional affiliations. 